\title{
Metastable behavior of premixed gas flames in rectangular channels
}

\author{
H. BERESTYCKI ${ }^{\dagger}$ \\ EHESS (École des hautes études en sciences sociales), CAMS, \\ 54 Boulevard Raspail, 75006 Paris, France \\ AND \\ L. KAGAN \\ School of Mathematical Sciences, Tel Aviv University, Ramat Aviv, Tel-Aviv 69978, Israel
}

[Received 9 September 2003 and in revised form 2 May 2004]

\begin{abstract}
A two-dimensional model for the upward propagating flame in a vertical square channel is explored. It is proved that under certain special initial conditions, the point where the flame interface attains its maximum, stays off the boundary (channel's wall) for an exponentially long period of time. The proof is an extension of the analysis developed previously for the one-dimensional version of the problem.
\end{abstract}

\section{Introduction and main results}

In our earlier works [5], [6], we studied a one-dimensional model for upward propagating flames. Our intention was to explain some of the metastable behavior.

In [6], the model was shown to reduce to the equation

$$
u_{t}-\varepsilon u_{x x}+u u_{x}-u=0 \quad \text { for } x \in(0,1), t>0
$$

together with

$$
\begin{gathered}
u(t, 0)=u(t, 1)=0, \\
u(0, x)=u_{0}(x) .
\end{gathered}
$$

In Section 3 below, we recall some of the main results from [6] concerning this equation.

In this paper, we address the problem in higher dimensions. From a mathematical point of view, the problem is more involved. We now describe our main results.

We consider the initial-boundary value problem

$$
\begin{aligned}
& \Phi_{t}-\frac{1}{2}|\nabla \Phi|^{2}=\varepsilon \Delta \Phi+\Phi-\langle\Phi\rangle \quad \text { in } S=\{(x, y, t):(x, y) \in D, t>0\} \\
& \Phi(0, x, y)=\Phi_{0}(x, y), \quad(x, y) \in D \\
& \frac{\partial \Phi}{\partial n}=0 \quad \text { on } \partial D \times(0, \infty) \\
& \langle\Phi\rangle=\int_{0}^{1} \int_{0}^{1} \Phi(t, x, y) \mathrm{d} x \mathrm{~d} y .
\end{aligned}
$$

†Email: hb@ehess.fr

‡Email: kaganleo@post.tau.ac.il

${ }^{\S}$ Corresponding author. Email: kamin@post.tau.ac.il

II Email: grishas@post.tau.ac.il 
In the one-dimensional case we set $u=-\partial \Phi / \partial x$, and arrive at (1.1). Here we study the case where $D$ is the rectangle $(0,1) \times(0,1)$ and $\Phi_{0} \in C(\bar{D})$.

Equation (1.1) contains a nonlocal term, but by the transformation

$$
\Phi \mapsto \widetilde{\Phi}=\Phi+e^{t} \int_{0}^{t} e^{-\tau}\langle\Phi\rangle \mathrm{d} \tau
$$

it reduces to

$$
\widetilde{\Phi}_{t}-\frac{1}{2}|\nabla \widetilde{\Phi}|^{2}=\varepsilon \Delta \widetilde{\Phi}+\widetilde{\Phi}
$$

Initial and boundary conditions are transformed into

$$
\begin{aligned}
& \widetilde{\Phi}(0, x, y)=\widetilde{\Phi}_{0}(x, y)=\Phi_{0}(x, y), \\
& \frac{\partial \widetilde{\Phi}}{\partial n}=0 \quad \text { on } \partial D \times(0, \infty) .
\end{aligned}
$$

The existence and uniqueness of the solution for the problem (1.5)-(1.7) is guaranteed by the standard theory of parabolic equations. By using the inverse transformation $\Phi=\widetilde{\Phi}-\int_{0}^{t}\langle\widetilde{\Phi}\rangle \mathrm{d} \tau$, we deduce that the solution of the problem (1.2)-(1.4) exists and is unique.

We analyze below the long-time dynamics of the solution $\Phi(t, x, y)$ when $\varepsilon>0$ is very small. Of particular interest is to describe the motion of the point $(x(t), y(t))=P(t)$ where $\Phi(t, \cdot)$ reaches its maximum. We prove that under some special assumptions on the initial data $\Phi_{0}$, the point $P(t)$ stays off $\partial D$ for an exponentially long time. Nevertheless as $t \rightarrow \infty$ the point $P(t)$ approaches one of the corners of $D$ and for $\varepsilon$ small enough the shape of $\Phi=\Phi_{\varepsilon}$ becomes close to a paraboloid. We call this phenomenon metastability. More specifically, the metastability is a process where the solution slowly evolves over an exponentially long time, provided the parameter $\varepsilon$ is close to zero.

We now formulate the assumptions on the initial function $\Phi_{0}(x, y)$ :

Assumption A1 There exist constants $a>0, b>0$ such that $a<b$ and

$$
\begin{array}{ll}
\frac{\partial \Phi_{0}}{\partial x}>0 & \text { for }(x, y) \in(0, a) \times[0,1], \\
\frac{\partial \Phi_{0}}{\partial x}<0 & \text { for }(x, y) \in(b, 1) \times[0,1] .
\end{array}
$$

Assumption A2 There exist constants $c>0, d>0$ such that $c<d$ and

$$
\begin{array}{ll}
\frac{\partial \Phi_{0}}{\partial y}>0 & \text { for }[0,1] \times(0, c), \\
\frac{\partial \Phi_{0}}{\partial y}<0 & \text { for }[0,1] \times(d, 1) .
\end{array}
$$

A paraboloid with its tip at the point $\left(x_{0}, y_{0}\right) \in(0,1) \times(0,1)$ is an example of a function which satisfies A1-A2.

Without loss of generality we also assume that $\Phi_{0}(x, y) \in C^{2}(\bar{D})$ and $\partial \Phi_{0} / \partial n=0$ on $\partial D$.

THeorem 1.1 Suppose $\Phi_{0}(x, y)$ satisfies A1-A2 and $\Phi=\Phi_{\varepsilon}(t, x, y)$ is the solution of (1.1)(1.3). Let $\delta$ be an arbitrary small number less than $\min \{a, 1-b, c, 1-d\}$. Then there are constants $\alpha>0$ and $\varepsilon_{0}>0$ such that for all $\varepsilon<\varepsilon_{0}$,

$$
-\frac{\partial \Phi_{\varepsilon}}{\partial x}<0 \quad \text { for }(x, y) \in(0, a-\delta) \times(0,1)
$$




$$
\begin{array}{ll}
-\frac{\partial \Phi_{\varepsilon}}{\partial x}>0 & \text { for }(x, y) \in(b+\delta, 1) \times(0,1), \\
-\frac{\partial \Phi_{\varepsilon}}{\partial y}<0 & \text { for }(x, y) \in(0,1) \times(0, c-\delta), \\
-\frac{\partial \Phi_{\varepsilon}}{\partial y}>0 & \text { for }(x, y) \in(0,1) \times(d+\delta, 1),
\end{array}
$$

for all $t$ with $0 \leqslant t \leqslant T_{\varepsilon}:=e^{\alpha / \varepsilon}$.

REMARK 1 Let $D_{1}$ be a rectangle $(a, b) \times(c, d)$. It follows from (1.8)-1.11 that the points of maximum of $\Phi_{\varepsilon}(t, x, y)$ for any fixed $t$ remain inside $D_{1}$ for an exponentially long time. This fact is an evidence of the metastable behavior of the solution.

REMARK 2 The values of $\alpha$ and $\varepsilon_{0}$ in Theorem 1.1 depend only on the values of $a, b, c, d$ and $\delta$.

Let $f_{\varepsilon}^{+}(x)$ be the unique positive solution of the ODE boundary value problem

$$
\left\{\begin{array}{l}
\varepsilon f^{\prime \prime}-f f^{\prime}+f=0 \quad \text { in }(0,1) \\
f(0)=f(1)=0
\end{array}\right.
$$

(see [6]).

TheOREM 1.2 Suppose $\Phi_{0}(x, y)$ satisfies A1-A2 and $b<1 / 2, d<1 / 2$. Then for all $\varepsilon$ sufficiently small

$$
\lim _{t \rightarrow \infty}\left[\Phi_{\varepsilon}(t, x, y)-\Phi_{\varepsilon}(t, 0,0)\right]=-\left(\int_{0}^{x} f_{\varepsilon}^{+}(\sigma) \mathrm{d} \sigma+\int_{0}^{y} f_{\varepsilon}^{+}(\sigma) \mathrm{d} \sigma\right) .
$$

The convergence in (1.13) is uniform in $D$.

REMARK 3 The geometrical meaning of Theorem 1.2 is that as $t \rightarrow \infty$ the shape of $\Phi(t, \cdot)$ tends to a bell with its tip at the corner $(0,0)$. The choice of the corner depends on the initial conditions $\Phi_{0}(x, y)$.

There are several papers dealing with metastable behavior for various physical problems. In the earlier works by Carr and Pego [9], Fusco and Hale [10] the Allen-Cahn equation is studied and the exponentially slow motion of the solution is proved. Some other papers deal with Allen-Cahn and Cahn-Hillard equations (see [1], [2], [4], [7], [12], [18], [19], [20]). The flame front model was considered by the present authors in [5], [6] and also by Sun and Ward in [17]. In [17] the formal asymptotic expansion for the movement of the solution is presented for a one-dimensional case.

For general surveys on metastability the reader may consult [19] and [20]. In particular, dynamics of the solution for the constrained Allen-Cahn equation is studied in [20] employing formal asymptotic approaches. The solution develops an internal layer which drifts exponentially slowly toward the closest point on the boundary and then slides along the boundary until it comes close to the point where the curvature has a local maximum.

A similar effect occurs for the flame model studied in the current paper. For a rectangular domain we prove that the point of maximum of the solution tends to one of the corners, and our numerical simulations show that this point of maximum first slowly approaches the closest point on the boundary and then drifts to the corner (Fig. (4). 
Such a behavior is different from the behavior of the solution for the so-called exit problem dealing with the Dirichlet boundary condition. As a result, for the exit problem the long time behavior of the solution is defined by the points of minimum of the potential on the boundary.

REMARK 4 In the proofs below it is essential that $D$ is a rectangle, which allows utilization of the results obtained for the one-dimensional case, studied previously. The case of a general domain remains an open problem.

\section{The physical model}

The current paper deals with the intrinsic dynamics of an upward propagating premixed gas flame spreading through a vertical square channel. In suitably chosen units the flame evolution is described by the model [1.1]-(1.3) involving only one equation and one parameter [13], [14]. Here $\Phi$ is the perturbation of the planar flame in units of $\gamma g L^{2} / 2 U_{b}^{2} ;(x, y, t)$ are the spatio-temporal coordinates in units of $L$ and $2 U_{b} / \gamma g L^{2}$, respectively; $\varepsilon=2 D_{M} U_{b} / \gamma g L^{2} ; L$ is the width of the channel; $U_{b}$ is the flame velocity relative to the burned gas; $g$ is the acceleration due to gravity; $\gamma=\left(\rho_{u}-\rho_{b}\right) / \rho_{u}$ is the thermal expansive parameter; $\rho_{u}, \rho_{b}$ are the densities of the unburned (cold) and burned (hot) gas, respectively; $D_{M}$ is the so-called Markstein diffusivity, assumed to be positive.

Equation (2.1) was derived within the framework of the Boussinesq-type model for flamebuoyancy interaction which neglects density variation everywhere but in the external forcing term [12]. The weakly nonlinear model described by equation 1.3 corresponds to the limit

$$
U_{b}^{2} / \gamma g L \gg 1,
$$

which is easily attainable in many realistic systems.

For example, at $L=5 \mathrm{~cm}, U_{b}=500 \mathrm{~cm} / \mathrm{s}, g=1000 \mathrm{~cm} / \mathrm{s}^{2}, \gamma=0.8$, the left-hand side of (2.1) amounts to 62.5 .

The previous studies of the problem dealt with a one-dimensional slab geometry, $0<x<L$. The results obtained may be briefly outlined as follows.

First, the problem (1.1)-1.3 admits a basic planar solution, $\Phi=0$, which, however, becomes unstable at $\varepsilon<\varepsilon_{0}=\pi^{-2} \approx 0.10$. At $\varepsilon \lesssim \varepsilon_{0}$ any initial perturbation rapidly leads to an equilibrium solution where the flame slope appears as a monotonic function of $\varepsilon$.

At $\varepsilon \ll \varepsilon_{0}$ (experimentally typical situation) the final result is qualitatively the same as for $\varepsilon \lesssim \varepsilon_{0}$. However, depending on the initial conditions, the character of the transient behavior may be markedly different for a rather wide class of initial data. At the early stage of its development, the solution is rapidly attracted to an intermediate state where the flame assumes a somewhat asymmetric parabolic shape. The subsequent evolution occurs at a slow rate which may become even extremely slow, provided $\varepsilon$ is small enough. In the course of this quasi-steady development, the tip of the parabola gradually moves towards one of the walls. As it comes close enough to the wall, the rate of flame evolution undergoes an abrupt increase. The final equilibrium state is formed when the tip touches the wall.

The current study deals with a two-dimensional version of the problem when the flame spreads through a square channel: $0<x<1,0<y<1$. Similarly to the one-dimensional case, at small $\varepsilon$ one can identify three stages of the flame evolution: (i) initial, when the flame rapidly assumes a shape of a somewhat shifted paraboloid, (ii) intermediate, when the flame evolution proceeds at a slow rate, and (iii) final, when the previously formed paraboloid rapidly reaches the ultimate equilibrium with its tip settling at one of the channel's edges. 

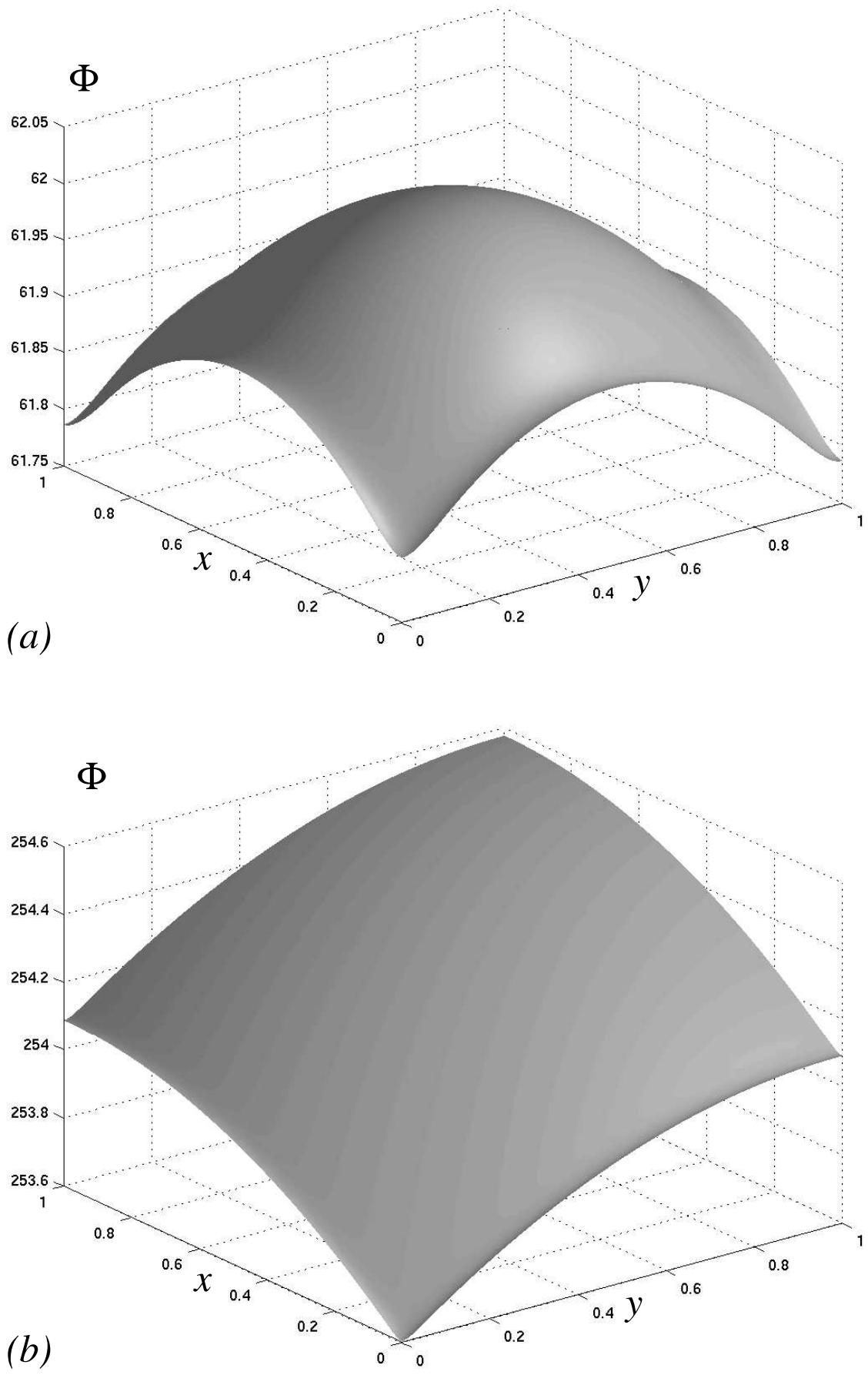

FIG. 1. Spatial configurations of the flame interface $\Phi(x, y, t)$ at two instants of time: $t=1000$ (a) and $t=2500$ (b). 

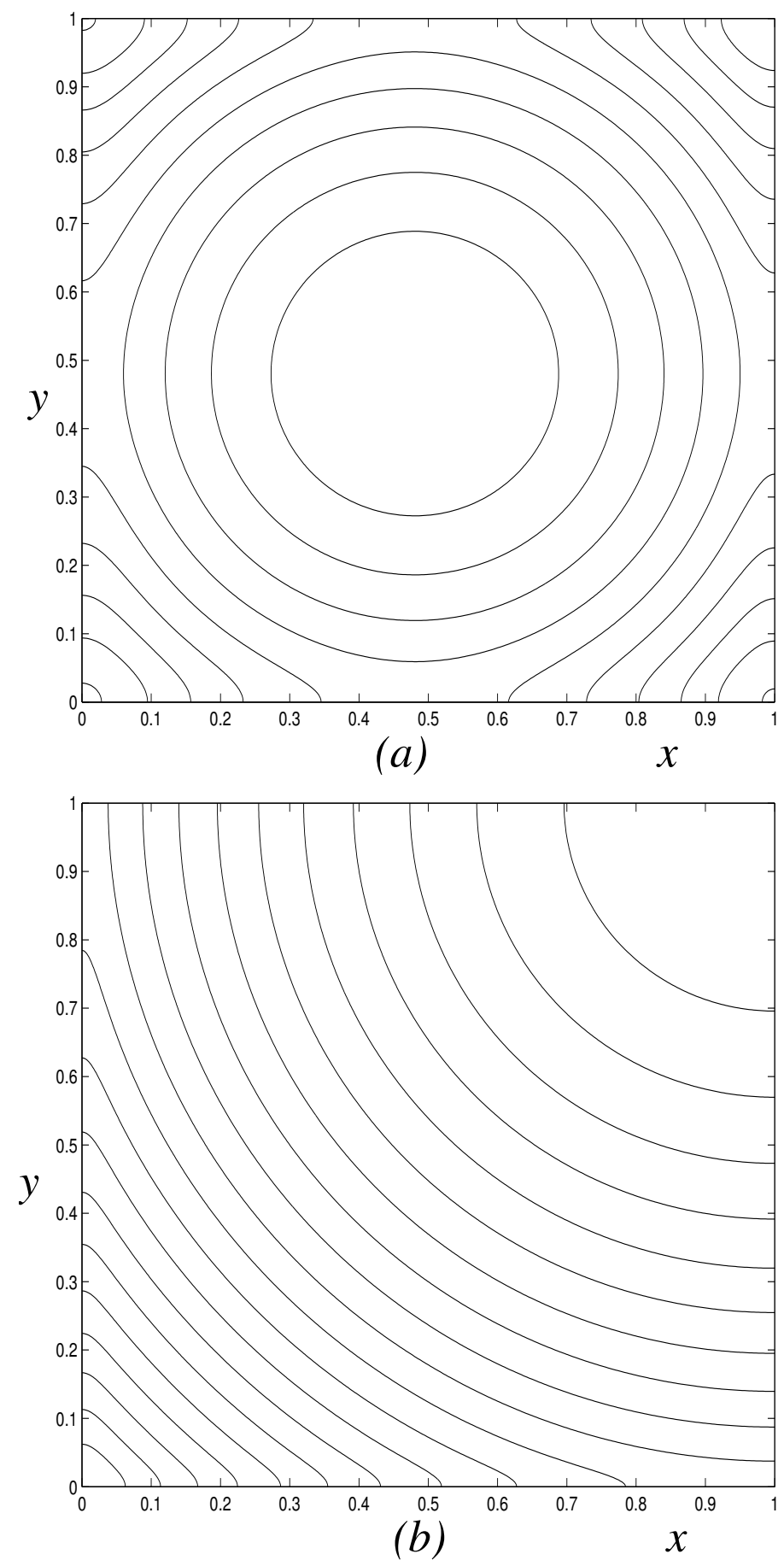

FIG. 2. Level sets of the flame interface $\Phi(x, y, t)$ at two instants of time: $t=1000$ (a) and $t=2500$ (b). 
When $\varepsilon=0$, equation (1.2) allows for a set of paraboloidal solutions,

$$
\Phi=V t+\Psi(x, y)
$$

with

$$
\Psi(x, y)=-\frac{1}{2}\left[\left(x-x_{0}\right)^{2}+\left(y-y_{0}\right)^{2}\right]
$$

and

$$
V=\frac{1}{3}-\frac{1}{2}\left[y_{0}\left(1-y_{0}\right)+x_{0}\left(1-x_{0}\right)\right]
$$

where $\left(x_{0}, y_{0}\right)$ is the location of the flame tip.

As is readily seen, $V=1 / 12$ if $x_{0}=y_{0}=1 / 2$, i.e. when the tip is at the channel's centerline, and $V=1 / 3$ if $x_{0}=y_{0}=0$, i.e. when the tip is at one of the channel's corners.

Figures 1 4 4 show results of numerical simulations of the problem (1.2)-(1.4) for $\varepsilon=0.01$ and $\Phi(x, y, 0)=\Psi(x, y)$. Two different initial conditions were employed: (a) the symmetry condition with a maximum located at $x_{0}=y_{0}=0.51$, and (b) the asymmetry condition with $x_{0}=0.51$ and $y_{0}=0.55$.

The spatial configurations of the flame surface are plotted in Figs. 1 and 2. (a) corresponds to the incipient time interval during which the changes take place only in the boundary layer according to condition (1.4). (b) shows the final well-settled configuration of the flame. Figs. 1 and 2 correspond to the symmetry case. For the asymmetry case the configurations are nearly identical.

The flame velocity is naturally defined as $V=\mathrm{d}\langle\Psi\rangle / \mathrm{d} t$. Its temporal evolution is shown in Fig. 3 Fig. 4 depicts trajectories of the maxima. One can see that in the case of symmetry the flame undergoes a single jump whereas in the case of asymmetry the flame jumps twice, first in the $y$ direction and then in the $x$ direction. In other words the flame is attracted to the nearest boundary ( $y=1$ in our simulation) and then to the nearest corner. Yet if the distances to both boundaries are equal the resulting velocity vector is directed straight to the corner.

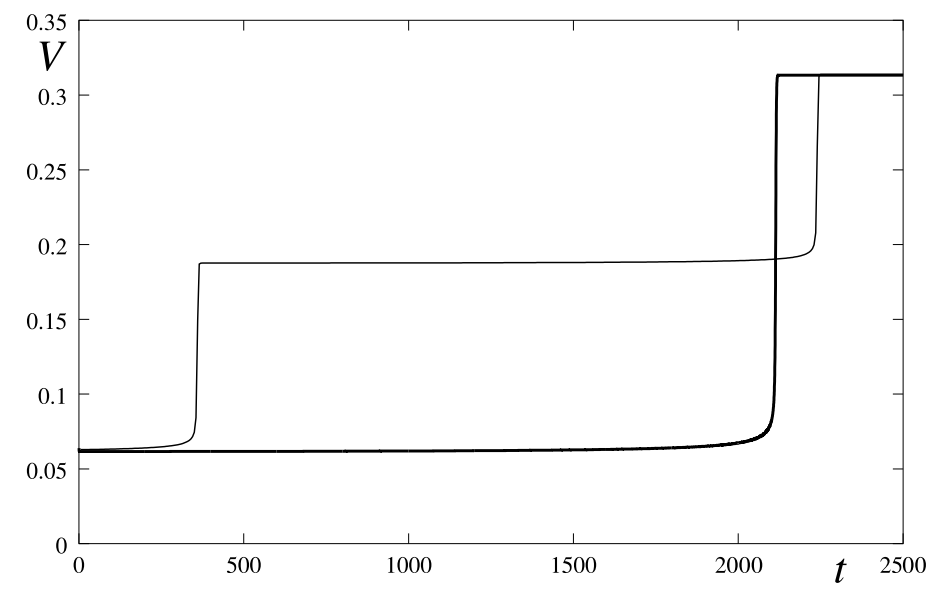

FIG. 3. Temporal evolutions of the flame velocity $V=\mathrm{d}\langle\Psi\rangle / \mathrm{d} t=\frac{1}{2}\left\langle(\nabla \Phi)^{2}\right\rangle$ for the symmetry (bold line) and asymmetry (thin line) cases. 


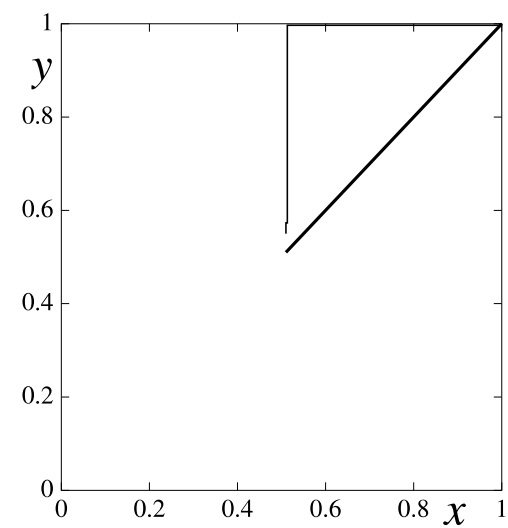

FIG. 4. Trajectories of the maxima for the symmetry (bold line) and asymmetry (thin line) cases.

\section{One-dimensional case revisited}

In the one-dimensional version of $(1.2)$,

$$
u=-\Phi_{x},
$$

one obtains the equation

$$
u_{t}-\varepsilon u_{x x}+u u_{x}-u=0 \quad \text { for } x \in(0,1), t>0,
$$

together with

$$
\begin{gathered}
u(t, 0)=u(t, 1)=0, \\
u(0, x)=u_{0}(x) .
\end{gathered}
$$

Note that the results obtained for equation $(3.1)$ can be immediately translated into those for the one-dimensional version of equation (1.2). It is worthwhile to keep in mind that when $u(t, \cdot)$ is close to linear, then $\Phi$ is close to a parabola. The tip of this parabola corresponds to the point where $u$ vanishes. In particular, when $u$ does not change sign, then $\Phi$ is monotonic and the tip of the parabola is at one of the endpoints. Equation (3.1) is of Burgers' type, but in contrast to the classical case, it contains a $-u$ term. It turns out that for the asymptotic behavior, this term plays an essential role.

Below we present some results from our previous paper [6] and the corollaries which we use for the two-dimensional case.

Let $f(x)$ be the stationary solution of 3.1 , 3.2), i.e. the solution of the ODE boundary value problem

$$
\begin{aligned}
& \varepsilon f^{\prime \prime}-f f^{\prime}+f=0 \quad \text { in }(0,1), \\
& f(0)=f(1)=0 .
\end{aligned}
$$

It is proved in [6] that for every $\varepsilon$ with $0<\varepsilon<\pi^{-2}$ there exists a unique positive solution $f_{\varepsilon}^{+}$. Likewise, there exists a unique negative solution $f_{\varepsilon}^{-}$. We call a solution $f$ positive (resp. negative) if $f(x)>0$ (resp. $f(x)<0$ ) for all $x \in(0,1)$. 
The next result is about the behavior of these solutions as $\varepsilon \rightarrow 0$. The solutions $f_{\varepsilon}^{+}$converge uniformly on compact sets of $[0,1)$ to the function $\varphi^{+}(x) \equiv x$ as $\varepsilon \rightarrow 0$. The solutions $f_{\varepsilon}^{-}$converge uniformly on compact sets of $(0,1]$ to the function $\varphi^{-}(x) \equiv x-1$ as $\varepsilon \rightarrow 0$. In [6] we consider the case when the initial function $u_{0} \in C_{0}^{0}[0,1]$ satisfies the condition:

$$
\begin{array}{ll}
u_{0}(x)<0 & \text { for all } x \in\left(0, a_{0}\right), \\
u_{0}(x)>0 & \text { for all } x \in\left(a_{0}, 1\right),
\end{array}
$$

where $a_{0}$ is some number between 0 and 1 .

Proposition 3.1 Suppose $u_{0}$ satisfies (3.5) and let $\delta$ be any small positive number less than $\min \left\{a_{0}, 1-a_{0}\right\}$. Then there are constants $\alpha>0$ and $\varepsilon_{0}>0$ such that for all $\varepsilon<\varepsilon_{0}$ and for all $0 \leqslant t \leqslant T_{\varepsilon}:=e^{\alpha / \varepsilon}$,

$$
\begin{array}{ll}
u_{\varepsilon}(t, x)>0 & \text { for all } x \in\left(a_{0}+\delta, 1\right), \\
u_{\varepsilon}(t, x)<0 & \text { for all } x \in\left(0, a_{0}-\delta\right) .
\end{array}
$$

Proposition 3.2 Suppose $u_{0}$ satisfies 3.5 . Fix some $\eta \in\left(0, \min \left\{a_{0}, 1-a_{0}\right\}\right)$ and let $\delta$ be any small positive number less than $\min \left\{a_{0}-\eta, 1-a_{0}-\eta\right\}$. Then there are constants $T>0, \alpha>0$ and $\varepsilon_{0}>0$ such that for all $\varepsilon<\varepsilon_{0}$,

$$
x-a_{0}-\delta \leqslant u_{\varepsilon}(t, x) \leqslant x-a_{0}+\delta
$$

for all $x \in[\eta, 1-\eta]$ and $T \leqslant t \leqslant T_{\varepsilon}:=e^{\alpha / \varepsilon}$.

For the proof of the statements mentioned above see [5, Theorems 1, 2, 4 and Proposition 8.1].

The last result from [6] we need is

PROPOSITION 3.3 Let $u_{0}$ satisfy condition 3.5 with $a_{0} \in(0,1 / 2)$. Then for $\varepsilon$ small enough $u_{\varepsilon}(t, x) \rightarrow f_{\varepsilon}^{+}(x)$ as $t \rightarrow \infty$. If $a_{0} \in(1 / 2,1)$ then $u_{\varepsilon}(t, x) \rightarrow f_{\varepsilon}^{-}(x)$. Moreover the convergence is uniform for $x \in[0,1]$.

For this last statement see [6, Theorem 5]. However this theorem states only the pointwise convergence and not uniform convergence. But the proof there is based on the uniqueness of the stationary solution and on the construction of super- and sub-solutions. Therefore uniform convergence follows (see [3], [15], [16]).

Now we generalize the previous statements in order to remove condition 3.5. The results we get are not as strong, but they apply to a much wider class of solutions.

The next assumption is the one-dimensional version of assumptions A1-A2:

Assumption A3 There exist constants $a>0, b>0$ such that $a<b$ and

$$
\begin{array}{ll}
u_{0}(x)<0 & \text { for } x \in(0, a), \\
u_{0}(x)>0 & \text { for } x \in(b, 1) .
\end{array}
$$

Suppose that $u_{0}$ satisfies A3 and let $\bar{u}_{0}$ be a continuous function defined on $[0,1]$ satisfying

$$
\begin{array}{ll}
\bar{u}_{0}(x) \geqslant u_{0}(x) & \text { for } x \in[0,1], \\
\bar{u}_{0}(x)<0 & \text { for } x \in(0, a), \\
\bar{u}_{0}(x)>0 & \text { for } x \in(a, 1),
\end{array}
$$


and

$$
\bar{u}_{0}(0)=\bar{u}_{0}(1)=0 .
$$

The existence of such a function for given $u_{0}$ is ensured by (3.9).

Similarly it follows from $\left(3.10\right.$ that there exists a continuous function $\underline{u}_{0}$ such that

$$
\begin{array}{ll}
\underline{u}_{0}(x) \leqslant u_{0}(x) & \text { for } x \in[0,1], \\
\underline{u}_{0}(x)<0 & \text { for } x \in(0, b), \\
\underline{u}_{0}(x)>0 & \text { for } x \in(b, 1), \\
\underline{u}_{0}(0)=\underline{u}_{0}(1)=0
\end{array}
$$

(see Fig. 5).

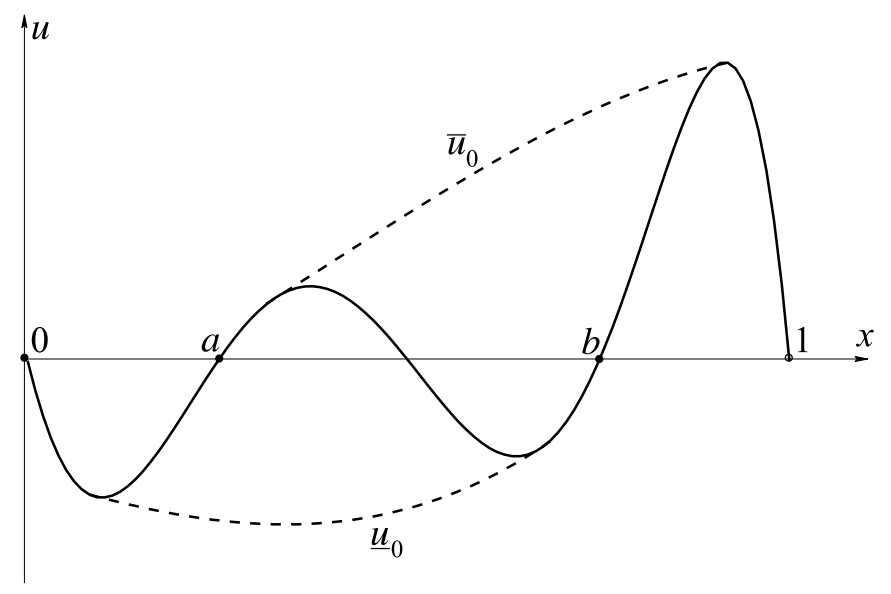

FIG. 5. Sketch illustrating the functions $\bar{u}_{0}$ and $\underline{u}_{0}$.

It is clear that both functions $\bar{u}_{0}$ and $\underline{u}_{0}$ satisfy $(3.5)$. Therefore the previously mentioned results may be applied. Now we are ready to prove the next

THEOREm 3.4 Suppose $u_{0}$ satisfies Assumption A3. Let $\delta$ be any small positive number less than $\min \{a, 1-b\}$. Then there are constants $\alpha>0$ and $\varepsilon_{0}>0$ such that for all $\varepsilon<\varepsilon_{0}$,

$$
\begin{array}{ll}
u_{\varepsilon}(t, x)<0 & \text { for } x \in(0, a-\delta), \\
u_{\varepsilon}(t, x)>0 & \text { for } x \in(b+\delta, 1),
\end{array}
$$

for all $0 \leqslant t \leqslant T_{\varepsilon}:=e^{\alpha / \varepsilon}$.

Proof. Let $\bar{u}_{0}(x)$ and $\underline{u}_{0}(x)$ be the functions satisfying conditions 3.11$)-(3.14)$ and 3.15 - 3.18$)$ respectively, and let $\bar{u}(t, x)$ (resp. $\underline{u}(t, x))$ be the solution of 3.1$)-(3.3)$ with initial data equal to $\bar{u}_{0}(x)\left(\operatorname{resp} . \underline{u}_{0}(x)\right)$. 
By the comparison principle using (3.11) and $(3.15)$ we obtain

$$
\underline{u}(t, x) \leqslant u_{\varepsilon}(t, x) \leqslant \bar{u}(t, x)
$$

for all $x \in[0,1]$ and $t \geqslant 0$. For $\bar{u}(t, x)$ we apply Proposition 3.6 and conclude that there are $\bar{\alpha}>0$ and $\bar{\varepsilon}_{0}>0$ such that for $\varepsilon \leqslant \bar{\varepsilon}_{0}$,

$$
\bar{u}(t, x)<0 \quad \text { for } x \in(0, a-\delta) \text { and } 0 \leqslant t \leqslant e^{\bar{\alpha} / \varepsilon} .
$$

Similarly, there are $\underline{\alpha}>0$ and $\underline{\varepsilon}_{0}>0$ such that for $\varepsilon \leqslant \underline{\varepsilon}_{0}$,

$$
\underline{u}(t, x)>0 \quad \text { for } x \in(b+\delta, 1) \text { and all } 0 \leqslant t \leqslant e^{\underline{\alpha} / \varepsilon} .
$$

The assertion of the theorem follows from 3.21 - 3.23 with $\varepsilon_{0}=\min \left(\bar{\varepsilon}_{0}, \underline{\varepsilon}_{0}\right), \alpha=\min (\bar{\alpha}, \underline{\alpha})$.

THEOREM 3.5 Suppose $u_{0}$ satisfies A3. Fix some $\eta \in[0, \min \{a, 1-b\}]$ and let $\delta$ be any positive number less than $\min \{a-\eta, 1-b-\eta\}$. Then there are constants $T>0, \alpha>0$ and $\varepsilon_{0}>0$ such that for all $\varepsilon<\varepsilon_{0}$,

$$
x-b-\delta<u_{\varepsilon}(t, x)<x-a+\delta \quad \text { for } x \in(\eta, 1-\eta) \text { and } T \leqslant t \leqslant T_{\varepsilon}:=e^{\alpha / \varepsilon} .
$$

Proof. Again use the solutions $\bar{u}(t, x), \underline{u}(t, x)$ and Proposition 3.2

Finally, we have

THEOREm 3.6 Suppose $u_{0}$ satisfies A3 and $b<1 / 2$. Then for all $\varepsilon$ sufficiently small,

$$
\lim _{t \rightarrow \infty} u_{\varepsilon}(t, x)=f_{\varepsilon}^{+}(x) \text {. }
$$

If $a>1 / 2$ then

$$
\lim _{t \rightarrow \infty} u_{\varepsilon}(t, x)=f_{\varepsilon}^{-}(x) .
$$

The convergence is uniform for $x \in[0,1]$.

Proof. This follows from (3.21) and Proposition 3.3.

\section{Two-dimensional case, rectangular channels}

In this section we consider the initial-boundary value problem

$$
\begin{aligned}
& \Phi_{t}-\frac{1}{2}|\nabla \Phi|^{2}=\varepsilon \Delta \Phi+\Phi-\langle\Phi\rangle \quad \text { in } S=\{(x, y, t):(x, y) \in D, t>0\}, \\
& \Phi(0, x, y)=\Phi_{0}(x, y), \quad(x, y) \in D, \\
& \frac{\partial \Phi}{\partial n}=0 \quad \text { on } \partial D \times(0, \infty),
\end{aligned}
$$

where $D$ is the rectangle $(0,1) \times(0,1)$ and $\Phi_{0} \in C(\bar{D})$.

Now we prove Theorems 1.1 and 1.2 formulated in the introduction. Let $v=-\partial \Phi / \partial x$. Differentiating (4.1] we obtain

$$
L v=v_{t}+v v_{x}+\frac{\partial \Phi}{\partial y} v_{y}-\varepsilon \Delta v-v=0 .
$$


It follows from 4.2 and 4.3 that

$$
\begin{aligned}
v(0, x, y) & =-\frac{\partial \Phi_{0}}{\partial x}, \\
v(t, 0, y) & =v(t, 1, y)=0, \quad \frac{\partial v}{\partial y}(t, x, 0)=\frac{\partial v}{\partial y}(t, x, 1)=0 .
\end{aligned}
$$

Equation 4.4 is not self-contained because of the term $\frac{\partial \Phi}{\partial y} v_{y}$. Therefore, in general, we consider the system of two equations, one for $v=-\partial \Phi / \partial x$ and one for $w=-\partial \Phi / \partial y$. But in the case of the rectangular domain $D$ we avoid this difficulty by using the following simple observation which is the key to the proofs of Theorems 1.1 and 1.2 .

Let $v$ be a solution of the problem (4.4) with the initial data which does not depend on $y$. Then $v$ stays independent of $y$ for all $t>0$.

LEMMA 4.1 Let $u(t, x)$ be the solution of the problem

$$
\begin{aligned}
& u_{t}+u u_{x}-\varepsilon u_{x x}-u=0 \\
& u(t, 0)=u(t, 1)=0, \\
& u(0, x)=u_{0}(x)
\end{aligned}
$$

Then $u(t, x)$ is a solution of the problem (4.4)-(4.6) which does not depend on $y$.

The proof is by substitution.

Now we consider $v(t, x, y)$ as a solution of the parabolic equation (4.4) in which $\partial \Phi / \partial y$ appears as a coefficient. Lemma 4.1 will be used for comparing solutions of (4.4)-(4.6) with solutions of the problem 4.7)-4.9.

LEMMA 4.2 (comparison) Let $v(t, x, y)$ be a solution of the problem 4.4 - 4.6, and $\underline{v}(t, x)$, $\bar{v}(t, x)$ be two solutions of the problem 4.7)-4.9. Suppose that

$$
\underline{v}(0, x) \leqslant v(0, x, y) \leqslant \bar{v}(0, x) \quad \forall(x, y) \in D .
$$

Then for all $t>0$,

$$
\underline{v}(t, x) \leqslant v(t, x, y) \leqslant \bar{v}(t, x), \quad x \in(0,1), y \in(0,1), t>0 .
$$

LEMMA 4.3 Let $\Phi_{\varepsilon}(t, x, y)$ be a solution of the problem 4.1 - (4.3) and suppose $\Phi_{0}(x, y)$ satisfies assumption A1. Let $v_{\varepsilon}=-\partial \Phi / \partial x$ and let $\delta$ be any small positive number less than $\min \{a, 1-b\}$. Then there are constants $\alpha>0$ and $\varepsilon_{0}>0$ such that for all $\varepsilon<\varepsilon_{0}$,

$$
\begin{array}{ll}
v_{\varepsilon}(t, x, y)<0 & \text { for all } x \in(0, a-\delta), y \in(0,1) \text { and } 0 \leqslant t \leqslant T_{\varepsilon}:=e^{\alpha / \varepsilon}, \\
v_{\varepsilon}(t, x, y)>0 & \text { for all } x \in(b+\delta, 1), y \in(0,1) \text { and } 0 \leqslant t \leqslant T_{\varepsilon} .
\end{array}
$$

Proof. Let $\underline{v}(t, x)$ be a solution of the problem 4.7)-4.9] with $\underline{v}(0, x)=\min _{y \in[0,1]} v(0, x, y)$ and $\bar{v}(t, x)$ be a solution of 4.7)-4.9] with $\bar{v}(0, x)=\max _{y \in[0,1]} v(0, x, y)$.

It follows from A1 that

$$
\underline{v}(0, x)<0, \quad \bar{v}(0, x)<0 \quad \text { for } x \in(0, a),
$$




$$
\underline{v}(0, x)>0, \quad \bar{v}(0, x)>0 \quad \text { for } x \in(b, 1) .
$$

Applying Theorem 3.4 to $\bar{v}(t, x)$ we deduce using 4.12 that for a fixed $\delta$ there exist $\widetilde{\alpha}$ and $\widetilde{\varepsilon}$ such that for all $\varepsilon<\widetilde{\varepsilon}$,

$$
\bar{v}(t, x) \leqslant 0 \quad \text { for all } x \in(0, a-\delta) \text { and } 0 \leqslant t \leqslant e^{\widetilde{\alpha} / \varepsilon} .
$$

By Lemma 4.2

$$
v_{\varepsilon}(t, x, y) \leqslant \bar{v}_{\varepsilon}(t, x),
$$

therefore with the same $\delta, \widetilde{\alpha}$ and $\widetilde{\varepsilon}$, for $\varepsilon<\widetilde{\varepsilon}$,

$$
v_{\varepsilon}(t, x, y) \leqslant 0 \quad \text { for all } x \in(0, a-\delta), y \in(0,1) \text { and } 0 \leqslant t \leqslant e^{\widetilde{\alpha} / \varepsilon} .
$$

Next we compare $v_{\varepsilon}(t, x, y)$ and $\underline{v}_{\varepsilon}(t, x)$. By Lemma 4.2 .

$$
v_{\varepsilon}(t, x, y) \geqslant \underline{v}_{\varepsilon}(t, x) .
$$

Applying Theorem 3.4 to $\underline{v}(t, x)$ we find that for a fixed $\delta$ there exist $\widehat{\alpha}$ and $\widehat{\varepsilon}$ such that for all $\varepsilon<\widehat{\varepsilon}$,

$$
\underline{v}_{\varepsilon}(t, x) \geqslant 0 \quad \text { for all } x \in(b+\delta, 1) \text { and } 0 \leqslant t \leqslant e^{\widehat{\alpha} / \varepsilon} .
$$

Consequently, with the same $\delta, \widehat{\alpha}$ and $\widehat{\varepsilon}$, for $\varepsilon<\widehat{\varepsilon}$,

$$
v_{\varepsilon}(t, x, y) \geqslant 0 \quad \text { for all } x \in(b+\delta, 1), y \in(0,1) \text { and } 0 \leqslant t \leqslant e^{\widehat{\alpha} / \varepsilon} .
$$

Finally, we choose $\alpha=\min (\widetilde{\alpha}, \widehat{\alpha}), \varepsilon_{0}=\min (\widetilde{\varepsilon}, \widehat{\varepsilon})$, and using 4.14, (4.15) we deduce the statement of the lemma.

Lemma 4.3 implies (1.8) and (1.9). In order to prove (1.10, 1.11 we define $w=-\partial \Phi / \partial y$ and proceed in the same way as with $v$. The function $w$ satisfies the equation

$$
L_{1} w=w_{t}+w w_{y}+\frac{\partial \Phi}{\partial x} w_{x}-\varepsilon \Delta w-w=0
$$

and

$$
\begin{aligned}
& w(0, x, y)=-\frac{\partial \Phi_{0}}{\partial y}, \\
& w(t, x, 0)=w(t, x, 1)=0, \quad \frac{\partial w}{\partial x}(t, 0, y)=\frac{\partial w}{\partial x}(t, 1, y)=0 .
\end{aligned}
$$

Next we compare $w_{\varepsilon}$ with the solutions of the one-dimensional problem 4.7)-4.9] where $x \rightarrow y$. First we define $\underline{w}(t, y)$ as a solution of the problem

$$
\begin{aligned}
& z_{t}+z z_{y}-\varepsilon z_{y y}-z=0, \\
& z(t, 0)=z(t, 1)=0, \\
& z(0, y)=z_{0}(y),
\end{aligned}
$$

where $\underline{w}(0, y)=\min _{x \in[0,1]} w(0, x, y)$. By the comparison principle

$$
\underline{w}_{\varepsilon}(t, y) \leqslant w_{\varepsilon}(t, x, y) .
$$


Let $\bar{w}_{\varepsilon}(t, y)$ be a solution of $\left.4.16-4.18\right)$ with $\bar{w}_{\varepsilon}(0, y)=\max _{x \in[0,1]} w(0, x, y)$. Then

$$
w_{\varepsilon}(t, x, y) \leqslant \bar{w}_{\varepsilon}(t, y) .
$$

Applying Theorem 3.4 to $\underline{w}_{\varepsilon}(t, y)$ and $\bar{w}_{\varepsilon}(t, y)$ we end up with the following lemma which is similar to Lemma 4.3 .

LEMMA 4.4 Let $\Phi_{\varepsilon}(t, x, y)$ be a solution of the problem 4.1 - 4.3 and suppose $\Phi_{0}(x, y)$ satisfies assumption A2. Let $w=-\partial \Phi / \partial y$ and let $\delta$ be any small positive number less than $\min \{c, 1-d\}$. Then there are constants $\alpha>0$ and $\varepsilon_{0}>0$ such that for all $\varepsilon<\varepsilon_{0}$,

$$
\begin{array}{ll}
w_{\varepsilon}(t, x, y)<0 & \text { for all } y \in(0, c-\delta), x \in(0,1) \text { and } 0 \leqslant t \leqslant T_{\varepsilon}:=e^{\alpha / \varepsilon}, \\
w_{\varepsilon}(t, x, y)>0 & \text { for all } y \in(d+\delta, 1), x \in(0,1) \text { and } 0 \leqslant t \leqslant T_{\varepsilon} .
\end{array}
$$

Theorem 1.1 now follows from Lemmas 4.3 and 4.4

Proof of Theorem 1.2 Consider again the solutions $\underline{v}_{\varepsilon}(x, t)$ and $\bar{v}_{\varepsilon}(x, t)$ which were used for the proof of Lemma 4.3 By Lemma 4.2 we have

$$
\underline{v}_{\varepsilon}(t, x) \leqslant v_{\varepsilon}(t, x, y) \leqslant \bar{v}_{\varepsilon}(t, x) .
$$

It follows from Theorem 3.6 that under the assumptions of Theorem 1.2 for all $\varepsilon$ sufficiently small,

$$
\underline{v}_{\varepsilon}(t, x) \rightarrow f_{\varepsilon}^{+}(x), \quad \bar{v}_{\varepsilon}(t, x) \rightarrow f_{\varepsilon}^{+}(x) \quad \text { as } t \rightarrow \infty .
$$

Therefore

$$
-\frac{\partial \Phi_{\varepsilon}}{\partial x}=v_{\varepsilon}(t, x, y) \rightarrow f_{\varepsilon}^{+}(x) \quad \text { uniformly as } t \rightarrow \infty
$$

In a similar way $-\partial \Phi_{\varepsilon} / \partial y \rightarrow f_{\varepsilon}^{+}(y)$ uniformly as $t \rightarrow \infty$, thus implying 1.13 .

\section{Concluding remarks}

The main result of our paper is that under the conditions of Theorem 1.1 the point of maximum of the solution $\Phi(t, \cdot)$ remains inside the rectangle $D_{1}$ for an exponentially long time. On the other hand by Theorem 1.2 if $D_{1}$ is inside one of the quarters of the rectangle $D$ the shape of $\Phi(t, \cdot)$ tends to a bell with its tip at one of the domain's corners.

In particular the conditions of Theorem 1.2 are met if the surface $\Phi(0, x, y)$ has the form of a bell with tip not at the center of the rectangle. For this case our numerical calculations show that the tip first moves very slowly to the closest point on the wall and then to the closest corner. If the distances to two walls are equal the movement is in the direction to the closest corner.

For the general domain the problem remains open. Yet, one may expect that the features identified in the present study will hold for the general case as well. In particular, for large time the shape of $\Phi(t, \cdot)$ should approach a bell with its tip at some point on the boundary where the curvature is positive.

We believe that one may study the local stability of the stationary solutions using the estimates of the pertinent eigenvalues. Yet, the global approach we use seems to be more effective, at least for the problem considered in this paper. 


\section{Acknowledgments}

These studies were supported in part by the German-Israeli Foundation under Grant No. 69515.10.01, the United States-Israel Binational Science Foundation under Grant No. 2002008, the Israel Science Foundation under Grant No. 67-01, and the European Community Program RTNHPRN-CT-2002-00274.

The authors are grateful to the referees whose comments prompted a significant improvement of the exposition of the paper.

\section{REFERENCES}

1. Alikakos, N. D., Bates, P. W., \& Fusco, G. Slow motion for the Cahn-Hilliard equation in one space dimension. J. Differential Equations 90 (1991), 81-135. Z Zbl 0753.35042 MR 1094451

2. Alikakos, N. \& Fusco, G. Slow dynamics for the Cahn-Hilliard equation in higher spatial dimensions: the motion of bubbles. Arch. Rat. Mech. Anal. 141 (1998), 1-61. Zbl 0906.35049 MR 1613496

3. Aronson, D. G., Crandall, M. G., \& Peletier, L. A. Stabilization of solutions of a degenerate nonlinear diffusion problem. Nonlinear Anal. 6 (1982), 1001-1022. Zbl 0518.35050 MR 0678053

4. BAtes, P. W. \& XUN, J. Metastable patterns for the Cahn-Hilliard equation: Parts I and II, J. Differential Equations 111 (1994), 421-457, Zbl 0805.35046 MR 1284421; J. Differential Equations 117 (1995), 165-216. Zbl 0805.35046 MR 1320187

5. Berestycki, H., Kamin, S., \& Sivashinsky, G. Nonlinear dynamics and metastability in a Burgers type equation (for upward propagating flames). C. R. Acad. Sci. Paris Sér. I 321 (1995), 185-190. Zbl 0838.35112 MR 1345444

6. Berestycki, H., Kamin, S., \& Sivashinsky, G. Metastability in a flame front evolution equation, Interfaces Free Bound. 3 (2001), 361-392. Zbl 0991.35097| MR 1869585

7. Bronsard, L. \& Hilhorst, D. On the slow dynamics for the Cahn-Hilliard equation in one space dimension. Proc. Roy Soc. London Ser. A 439 (1992), 669-682. Zbl 0777.35007 MR 1196438

8. BRONSARD, L. \& KoHn, R. V. On the slowness of phase boundary motion in one space dimension. Comm. Pure Appl. Math. 43 (1990), 983-998. Zbl 0761.35044 MR 1075075

9. CARr, J. \& Pego, R. Metastable patterns in solutions of $u_{t}=\varepsilon^{2} u_{x x}-f(u)$. Comm. Pure Appl. Math. 42 (1989), 523-576. Zbl 0685.35054 MR 0997567

10. Fusco, G. \& Hale, J. K. Slow motion manifolds, dormant instability and singular perturbations. $J$. Dynam. Differential Equations 1 (1989), 75-94. Zbl 0684.34055 MR 1010961

11. Grant, Ch. P. Slow motion in one-dimensional Cahn-Morral systems. SIAM J. Appl. Math. 26 (1995), 21-34. Zbl 0813.35042 MR 1311880

12. Laforgue, J. G. \& O'MALley, R. E. Shock layer movement of Burgers equation. SIAM J. Appl. Math. 55 (1995), 332-348. Zbl 0820.35119 MR 1322763

13. Mikishev, A. B. \& Sivashinsky, G. I. Quasi-equilibrium in upward propagating flames. Phys. Lett. A 175 (1993), 409-414. MR 1215405

14. RAKib, Z. \& Sivashinsky, G. I. Instabilities in upward propagating flames. Combust. Sci. Technol. 54 (1987), 69-84.

15. Sattinger, D. H. Monotone methods in nonlinear elliptic and parabolic boundary value problems. Indiana Univ. Math. J. 21 (1972), 979-1000. Zbl 0223.35038 MR 0299921

16. Smoller, J. Shock Waves and Reaction-Diffusion Equations, Springer (1983). Zbl 0508.35002 MR 0688146

17. SUN, X. \& WARD, M. J. Metastability for a generalized Burgers equation with applications to propagating flame-fronts. European J. Appl. Math. 10 (1999), 27-53. Zbl 0919.35122 MR 1685819 
18. SUN, X. \& WARD, M. Dynamics and coarsening of interfaces for the viscous Cahn-Hilliard equation in one spatial dimension, Stud. Appl. Math. 105 (2000), 203-234. Zbl pre01844273 MR 1783547

19. WARD, M. Exponential asymptotics and convection-diffusion-reaction models. Analyzing Multiscale Phenomena Using Singular Perturbation Methods, J. Cronin and R. O’Malley (eds.), Proc. Sympos. Appl. Math. 56, Amer. Math. Soc. (1998), 151-184. MR 1718885

20. WARD, M. J. Metastable dynamics and exponential asymptotics in multi-dimensional domains. Multiple Time-Scale Dynamical Systems, IMA Vol. Math. Appl. 122, C. K. R. T. Jones and A. Khibnik (eds.), Springer (2000), 233-260. Zbl 1007.35002 MR 1846579

21. WARD, M. J. \& REYNA, L. G. Internal layers, small eigenvalues and the sensitivity of metastable motion. SIAM J. Appl. Math. 55 (1995), 425-445. Zbl 0818.35008 MR 1322768 